\title{
A Software-Defined Channel Sounder for Industrial Environments with Fast Time Variance
}

\author{
Niels Hendrik Fliedner, Dimitri Block, Uwe Meier \\ inIT - Institute of Industrial Information Technologies \\ OWL University of Applied Sciences \\ Lemgo, Germany \\ Email: \{niels.fliedner, dimitri.block, uwe.meier\}@hs-owl.de
}

\begin{abstract}
Novel industrial wireless applications require wideband, real-time channel characterization due to complex multipath propagation. Rapid machine motion leads to fast time variance of the channel's reflective behavior, which must be captured for radio channel characterization. Additionally, inhomogeneous radio channels demand highly flexible measurements. Existing approaches for radio channel measurements either lack flexibility or wide-band, real-time performance with fast time variance.

In this paper, we propose a correlative channel sounding approach utilizing a software-defined architecture. The approach enables real-time, wide-band measurements with fast time variance immune to active interference. The desired performance is validated with a demanding industrial application example.
\end{abstract}

\section{INTRODUCTION}

The recent increase in performance requirements for devices in Industry 4.0 wireless applications demands the development of new wireless communication systems (WCSs) [1]. Such developments require channel characterization in areas previously not fully investigated [2].

Compared to office and outdoor environments, industrial environments behave drastically worse. They are ranging from highly absorbent to highly reflective environments [3]. Reflections cause multi-path propagation, which increase frequency variance in factory automation (FA) environments [4]. Multipath propagation leads to radio channel time dispersion causing inter-symbol interference (ISI) in WCS [2]. Characterization of channels with multi-path propagation requires wideband radio channel measurements.

Further, non-stationary multi-path channel conditions also impact FA environments WCS [4]. Especially rapid machine motion causes fast time variance of the channel's reflective behavior. This challenges time-critical closed-loop Industry 4.0 applications with latencies below $1 \mathrm{~ms}[1]$, [2]. In order to address those fast changes, the radio channel measurement demands to be fast.

Additionally, industrial environments are inhomogeneous, which ranges from highly reflective, complex machine cells to uniform storage areas and transportation paths. Such inhomogeneity leads to high spatial diversity within FA environments [3]. Spatial diversity requires detailed spatial channel characterization, and therefore dense spatial channel measurements. Thus, for FA environments the measurement methodology has to be flexible, scalable and ideally embedded in a low-cost hardware.
Few publications have shown implementation of real-time channel sounding with correlative sequences. [5] use softwaredefined radios (SDRs) with National Instruments' LabVIEW to program the correlation function directly into the fieldprogrammable gate array (FPGA) for enhanced performance. Any changes to the measurement setup have to be applied separate from the measurement via FPGA programming, which limits the flexibility. The hardware-defined HIRATE channel sounder introduced by [6] and utilized by [2] shows even better performance, but conversely is more limited to changes during measurement than [5]. To the best of our knowledge, so far no publication implements a software-defined real-time architecture for industrial radio channel measurements.

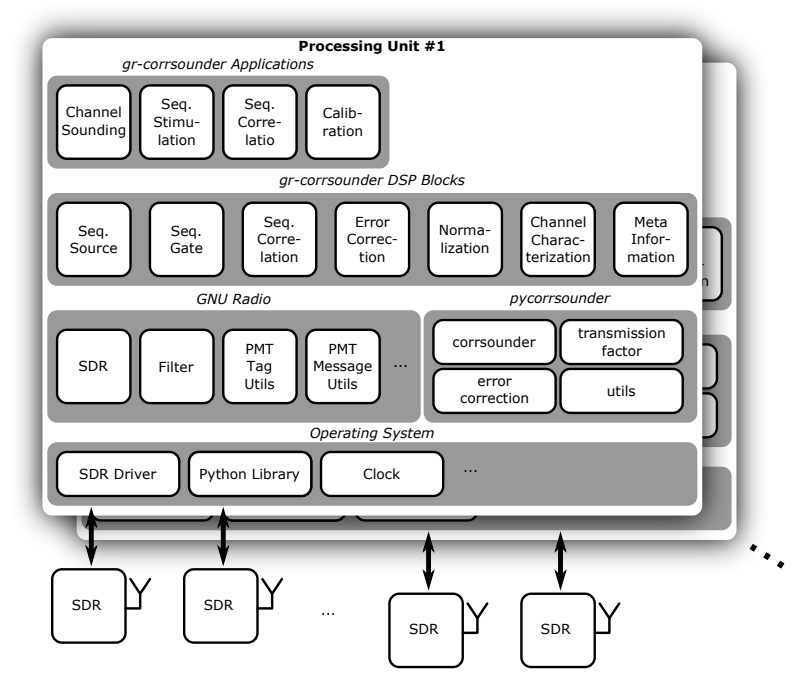

Fig. 1. Flexible and scalable architecture of the software-defined wireless channel sounding approach corrsounder for industrial real-time channel characterization

In this paper we propose a novel software-defined wireless channel sounding approach named corrsounder for industrial real-time channel characterization. In general, channel sounding techniques enable wide-band channel measurements, and therefore enable multi-path propagation characterization. While channel sounding suffers from limited accuracy, its periodic sequence-based stimulation enable the characterization of fast varying environments. Additionally, the software-defined realization enables flexible and scalable implementation with 
off-the-shelf radio frequency (RF) front ends as shown in Fig. 1. Furthermore, the proposed real-time capability allows for embedding the measurement system into live processing on demand.

This paper is structured as follows: Section II provides an overview of current solutions for industrial wireless channel sounding approaches, Section III describes the the mathematical basics of the utilized correlative channel sounding, Section IV details the proposed software architecture of corrsounder, Section $\mathrm{V}$ validates the desired performance with an application example, and finally a conclusion is drawn in Section VI

\section{INDUSTRIAL WIRELESS CHANNEL SOUNDING}

For channel measurements there exist three methods of channel stimulation: continuous-wave (CW), impulse-based and sequence-based stimulation. But $\mathrm{CW}$ - and impulse-based channel characterizations depend on emission measurements. Hence, they are sensitive to interference. Therefore, using these methods it is not possible to measure only the frequency or impulse response of an active industrial environment.

Sequence-based stimulations overcome the time limitation and take advantage of the repetitive structure. Thereby, various sequences exist for stimulation. For reception, sequencebased stimulation require correlation methods, which directly return the scaled impulse response. These correlation methods suppress the impact of any active interference dissimilar to the utilized sequence. In contrast to $\mathrm{CW}$ - and impulse-based stimulations, these methods enable the segregated measurement of the frequency or impulse response of an industrial environment with additional transmitters, which is called an active environment.

Generally speaking, correlative sequences can be differentiated into binary and non-binary ones. Typical binary sequences are pseudo-noise (PN) sequences. In general, binary sequences suffer from an inconstant spectral envelope, and therefore linear distortion. They are commonly realized in the form of maximum length sequences (MLSs), which offer the highest sequence length $N_{\text {seq }}$ at a certain linear-feedback shift register (LFSR) length $l$, with $N_{\text {seq }}=2^{l}-1$. The periodic autocorrelation function (PACF) of MLSs have a non-zero outof-phase value $v_{\text {oop }}=-1$ [9], and thus add a systematic error to the correlation, which limits the dynamic range to $D_{\mathrm{r}}=N_{\text {seq }}-\left|v_{\text {oop }}\right|$ [9]. Hence, they are not considered perfect sequences. MLSs provide a constant magnitude, and hence a peak-to-average-power ratio (PAPR) of one. This lowers the requirements on the automatic gain control (AGC) of RF frontends, and therefore SDRs.

For non-binary sequences, there are perfect ones which provide the desired property of a zero out-of-phase PACF value and therefore leads to the full dynamic range of $D_{\mathrm{r}}=$ $N_{\text {seq. }}$. An optimal choice for a non-binary perfect sequence is called Frank Zadoff Chu (FZC) sequence [10]. They also provide a constant magnitude, and hence a PAPR of one [11]. Additionally, the spectral envelope of FZC sequences is constant without linear distortion. Furthermore, these sequences are invulnerable to carrier frequency offset [11], which allows more flexible radio channel measurements in complex scenarios, since no further frequency synchronizing is needed. Additionally, multiple FZC same-length sequences can be generated, which enables multiple-input and multipleoutput (MIMO) channel measurement [12] and multi-user time synchronization, e.g. in 3GPP long-term evolution (LTE) communication [13].

\section{SySTEM DESCRIPTION}

The sequence-based sounding signal flow is illustrated in Fig. 2

For the time-discrete channel stimulation $x[t]$, a signal source outputs infinite repetitions of a chosen correlative sequence $x_{\text {seq }}[t]$ :

$$
x[t]=\sum_{i=-\infty}^{\infty} x_{\mathrm{seq}}\left[t-i T_{\mathrm{seq}}\right]
$$

with the duration $T_{\text {seq }}=T_{\mathrm{s}} \cdot N_{\text {seq }}$, with the sampling time $T_{\mathrm{s}}$ and the length $N_{\text {seq }}$. Then, the resulting repetitive signal is transmitted with a SDR.

The transmitting and receiving SDRs involve in-phase and quadrature (IQ) modulation and demodulation, respectivly. Additionally, they perform frequency translation. Hence, the SDRs handle the low-pass equivalent of a band-pass signal. So, a band-pass radio channel is stimulated and therfore also evaluated.

The radio channel influences the transmitted signal:

$$
y[t]=x[t] * h[t]+n
$$

with the equivalent low-pass impulse response $h[t]$ and additive white Gaussian noise (AWGN) $n$. It is important to note, that $h[t]$ contains the impact of the radio channel $h_{\mathrm{ch}}[t]$ and also the linear distortion caused by utilized antenna equipment such as cables $h_{\text {cable }}[t]$ :

$$
h[t]=h_{\mathrm{ch}}[t] * h_{\text {cable }}[t]
$$

The following signal processing block, called sequence gate, ensures the repetitive structure of the received signal $y[t]$ for correlation later-on. The repetitions may be disrupted by trigger events such as reception buffer overflow or from other external sources e.g. global navigation satellite system (GNSS) localization events. The sequence gate mutes disrupted sequence durations and passes only full ones. Its output signal can be expressed as:

$$
y_{\mathrm{g}}[t]=\sum_{j=-\infty}^{\infty} y\left[t-k_{j} T_{\text {seq }}\right] \cdot w_{\text {rect }}\left(\left(t-k_{j} T_{\text {seq }}\right) / T_{\text {seq }}\right)
$$

with the strictly monotonous growing index $k_{j}>k_{j-1}$ and the rectangular windowing function $w_{\text {rect }}(t / T)$, which is one for the time interval $[0, T)$ and otherwise zero. With no trigger events, $y_{\mathrm{g}}[t]$ is equal to $y[t]$. 


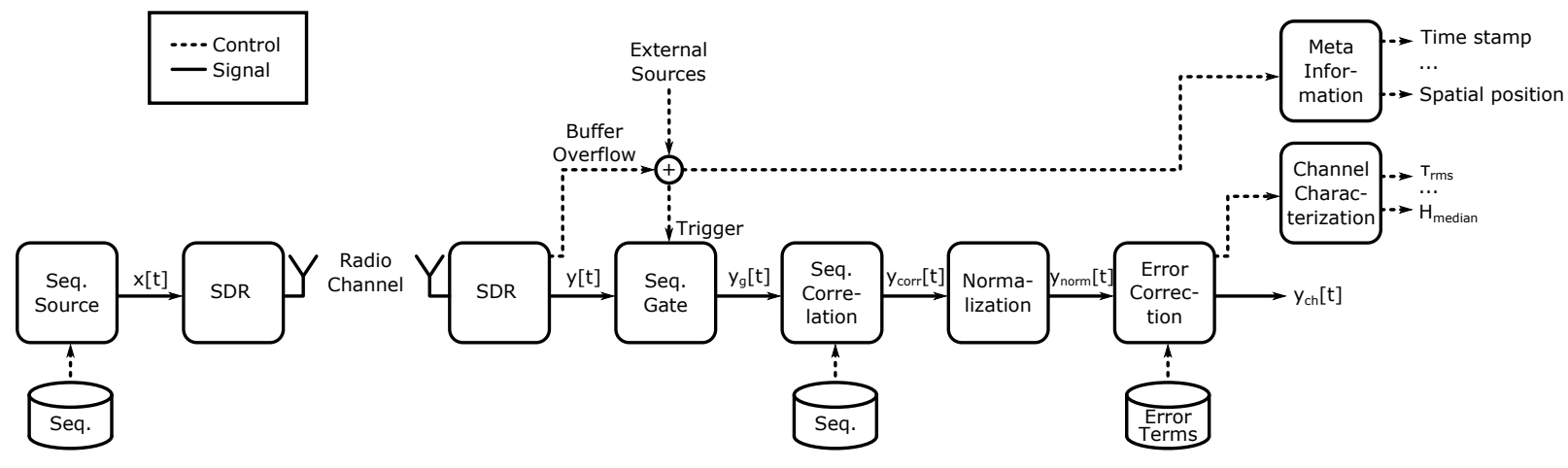

Fig. 2. Signal and control flow graph of the sequence-based channel sounding approach

Then, the gated signal $y_{\mathrm{g}}[t]$ is correlated with the chosen stimulation sequence $x_{\text {seq }}[t]$, which can be expressed as conjugate complex time-reversed convolution:

$$
y_{\text {corr }}[t]=y_{\mathrm{g}}[t] * x_{\mathrm{seq}}^{*}\left[-\left(t-T_{\mathrm{seq}}\right)\right]
$$

With no trigger events, $y_{\text {corr }}[t]$ can be separated into a noiseimpact term $y_{\text {corr, } \mathrm{n}}[t]$ and a signal term $y_{\text {corr,s }}[t]$ :

$$
\begin{aligned}
y_{\text {corr }}[t]= & (x[t] * h[t]) * x_{\mathrm{seq}}^{*}\left[-\left(t-T_{\mathrm{seq}}\right)\right] \\
& +n * x_{\mathrm{seq}}^{*}\left[-\left(t-T_{\mathrm{seq}}\right)\right] \\
= & y_{\text {corr, } \mathrm{s}}[t]+y_{\text {corr, } \mathrm{n}}[t]
\end{aligned}
$$

With good AWGN cross-correlation properties of the chosen stimulation sequence as discussed in Section III the impact of $y_{\text {corr, }}[t]$ can be neglected. Then, the associative property of convolution is applied:

$$
y_{\text {corr }}[t]=(\underbrace{x[t] * x_{\text {seq }}^{*}\left[-\left(t-T_{\text {seq }}\right)\right]}_{\text {PACF }}) * h[t]
$$

The PACF property of correlative sequences with constant outof-phase PACF value $v_{\text {oop }}$ results in:

$$
y_{\text {corr }}[t]=\left(v_{\text {oop }}+\left(N_{\text {seq }}-v_{\text {oop }}\right) \sum_{i=-\infty}^{\infty} \delta\left[t-t_{i}\right]\right) * h[t]
$$

with the measurement time substitution $t_{i}=(i+1) T_{\mathrm{seq}}-T_{\mathrm{s}}$.

Next, the distributive property of convolution and the sifting property of the Dirac function are applied. The result is a timeshifted and value-scaled impulse response series.

$$
\begin{aligned}
y_{\text {corr }}[t]= & \left(N_{\text {seq }}-v_{\text {oop }}\right) \sum_{i=-\infty}^{\infty} h\left[t-t_{i}\right] \\
& +v_{\text {oop }} * h[t]
\end{aligned}
$$

Hence, a non-zero $v_{\text {oop }}$ adds a bias error $v_{\text {oop }} * h[t]$, which is the case for non-perfect sequences.

In order to return the desired impulse response $h[t]$, the signal $y_{\text {corr }}[t]$ needs to be scaled according to the number of samples per sequence $N_{\text {seq }}$.

$$
y_{\text {norm }}[t]=\frac{1}{N_{\text {seq }}} \cdot y_{\text {corr }}[t]
$$

This also shows, that the bias error $v_{\text {oop }} * h[t]$ gets reduced with increased sequence length $N_{\text {seq }}$.

The forward transmission tracking error terms are used to correct the linear distortion caused by $h_{\text {cable }}[t]$, which is still contained within $y_{\text {norm }}[t]$. This correction is executed by convolution with $h_{\mathrm{ftt}}[t]$.

$$
\begin{aligned}
y_{\mathrm{ch}}[t] & =y_{\text {norm }}[t] * h_{\mathrm{ftt}}[t] \\
& \approx \sum_{i=-\infty}^{\infty} h_{\mathrm{ch}}\left[t-t_{i}\right]=\sum_{i=-\infty}^{\infty} h_{\mathrm{ch}}\left[\tau, t_{i}\right]
\end{aligned}
$$

with the finely granulated impulse response time $\tau$.

Thus, the proposed measurement method returns the soughtfor channel impulse response series.

\section{Software Architecture}

The channel sounding approach corrsounder is realized based on a modular software architecture. In the following subsections the software architecture shown in Fig. 1 is discussed.

\section{A. Distributed Processing}

Channel sounding with sequence-based stimulations can be splitted into the transmission part for stimulation and the reception part for correlation. These are independent from each other as shown in Fig. 2, as long as an identical sequence is provided. Hence, channel sounding stimulation and correlation may be separated, and the associated SDRs can be connected to multiple processing units such as embedded host computers. Each processing unit has to be connected to at least one SDR, performing either the stimulation or correlation processing. With multiple SDRs it may perform any arbitrary combination of the stimulation or correlation processing. Therefore, the software architecture approaches a distributed processing. It also enables integration into existing utilized wireless infrastructure, and therefore increases flexibility.

\section{B. Operating System}

The corrsounder expects a Linux-based operating systems (OSs) for the processing units. Hence, general-purpose Ubuntu OS for a high-power x86 hardware architecture, but also low-power embedded ARM-based Linux derivates for single-board computer (SBC) can be addressed. The OSs have 
to provide real-time hardware bindings to the SDRs such as device drivers. In case of additional trigger sources, the bindings such as hardware clocks and GNSS receivers also have to be provided by the OS . Additionally, the OS has to provide the Python runtime and the computation library numpy for signal processing.

\section{Kernel Module}

The kernel module pycorrsounder contains basic routines for channel sounding operation as well as for evaluation. It only requires the Python related libraries. Thus, the dependencies of corrsounder for pre- and post-processing are limited up to the kernel module, which increases the flexibility. pycorrsounder contains the following routines:

a) Sequence Generation and Correlation: Generation routines are provided for binary MLS and non-binary FZC sequences. Additional sequence generation routines can be added later on. The module also contains routines for sequence correlation: auto-correlation function (ACF), PACF, crosscorrelation function (CCF), and periodic cross-correlation function (PCCF). The ACF can be used for evaluation of a finite signal, while PACF is the counterpart for assumed infinite repetition of the finite signal. The CCF and PCCF can be used accordingly for evaluation of two non-equal signals, such as a transmitted sequence and the corresponding received channel response.

b) Error Correction and Filtering: For two-port systematic linear error correction with two SDR antenna ports, a through adjustment routine is provided. It requires the error term for forward transmission tracking. The error term can be acquired by directly connecting the SDR antenna ports. Through adjustment is easy to perform, removes the main frequency response error and can be utilized when medium accuracy is required. Various other distortion effects can be suppressed by the provided discrete filtering routines. A removal routine for peaks and a down-sampling routine are provided. The removal routine can suppress spectral and temporal peaks such as the direct current (DC) bias. The bias is caused by the local oscillator (LO) RF leakage of a receiving SDR [14]. It results in a constant value offset and therefore a spectral peak around $0 \mathrm{~Hz}$. Hence, the removal routine fades out such spectral frequency range and interpolates the values afterwards. The down-sampling routine is utilized to suppress high-frequency attenuation by extracting the low-frequency part. The routine determines a cutoff frequency at which the power spectral density (PSD) falls below a threshold relative to the maximum, e.g. $-3 \mathrm{~dB}$ for the edge frequency.

c) Channel Characterization: Routines are provided for channel characterization. Thereby, impulse response time $\tau$ and measurement time $t$ evaluation routines are provided for single-sequence and inter-sequence characterization, respectively. The former characterize the frequency and the impulse response at a certain measurement time $t$. Thereby, the power delay profile (PDP) $E\left[|h(\tau)|^{2}\right]$, the mean delay $\bar{\tau}$ and the delay spread $\tau_{\text {rms }}$ can be characterized. For the frequency response, the routines characterize the PSD $E\left[|H(f)|^{2}\right]$ and statistical metrics like percentile $10 \% H_{10}, 90 \% H_{90}, 50 \% H_{\text {median }}$ of the magnitude $|H(f)|$ and the coherence bandwidth $B_{C}$. Additionally, arbitrary distributions can be estimated like the Rician distribution for line-of-sight (LOS) conditions. Furthermore, the path-loss exponent (PLE) can be estimated based on the transmission and reception antenna gain. Measurement time characterization routines include for example the Doppler spread $h\left[\tau, f_{d}\right]$ and the coherence time $T_{C}$.

\section{GNU Radio}

In case the processing unit has to perform real-time processing, the well-known SDR framework GNU Radio is required. GNU Radio is a library of digital signal processing (DSP) blocks, SDR bindings and application routines with several command-line and graphical user interfaces. It is utilized in several simulation studies as well as for DSP application deployment. For the signal and control flow Fig. 2 different GNU Radio approaches are applied. For the signal flow, streambased items of complex IQ samples have to be utilized. GNU Radio provides in-tree DSP blocks for stream processing such as SDR source and sink, convolutional and DFT-based filter blocks. In contrast, the control flow can also be event-based for triggers. Therefore, GNU Radio provides in-tree blocks for event-based processing with polymorphic type (PMT) tags and PMT messages. PMT tags are synchronous events which annotate signal streams. PMT messages are asynchronous events which are processed concurrently and independent of the signal streams.

\section{E. Corrsounder DSP Blocks}

The corrsounder DSP blocks are provided within the out-of-tree (OOT) GNU Radio module gr-corrsounder. It requires a GNU Radio instance and the routines of the kernel module pycorrsounder. gr-corrsounder includes the DSP blocks sequence source, sequence gate, sequence correlation, error correction, channel characterization, and the meta information. These blocks are already discussed in Section III

\section{F. corrsounder DSP Applications}

The module gr-corrsounder also contains the corrsounder DSP applications. These are executable flow graphs of connected DSP blocks. Mainly, four applications are provided. The first application is called Channel Sounding. It represents the channel sounding signal and control flow shown in Fig. 2. It combines two applications: Sequence Stimulation and Sequence Correlation. Sequence Stimulation is an application for the stimulation part with the transmissionrelated processing blocks. Sequence Stimulation is the counterpart for reception-related part. The final application is called Calibration. It is a derivate of the Channel Sounding application without the error correction blocks. It has to be utilized for calibration to determine the error terms.

\section{G. Source Code Availability}

The source code of the channel sounder corrsounder is online available via the URL https://github.com/inIT-HF/ 
gr-corrsounder. It contains the module gr-corrsounder with the DSP blocks and applications as well as the kernel module pycorrsounder.

\section{Application Example}

An exemplary measurement demonstrates the performance of our proposed channel sounder architecture. The radio channel consists of a robot arm performing a pick-and-place routine inside a separated cell. Two antennas are placed in opposite corners with a distance of $3.8 \mathrm{~m}$ and a height of $1.5 \mathrm{~m}$. The robot arm obstructs the LOS periodically during motion.

We utilize distributed processing, with a vector signal generator (VSG) for stimulation and a host computer connected to an SDR for correlation. The stimulation sequence is generated by the kernel module pycorrsounder. For correlation, the corrsounder DSP application Sequence Correlation is used.

TABLE I

MeAsurement SETUP AND Parameters

\begin{tabular}{ll}
\hline Unit & Value \\
\hline VSG & Rohde\& Schwarz SMBV100A \\
Antennas & WiMo 18720.3H 5 GHz \\
Antenna gain & $5 \mathrm{dBi}$ \\
Robot arm & Reis RV16 \\
SDR & Ettus Research USRP X300 \\
Host computer OS & Ubuntu Linux 14.04 \\
\hline Center frequency & $5.80 \mathrm{GHz}$ \\
Sampling rate & $100 \mathrm{MSps}$ \\
Sequence type & FZC \\
Sequence length & $1024 \mathrm{samples}$ \\
FZC parameter & 7 \\
Measurement $3 \mathrm{~dB}$ bandwidth & $93.07 \mathrm{MHz}$ \\
Calibration routine & Through \\
DC bias suppression bandwidth & $781 \mathrm{kHz}$ \\
Measurement duration & $12 \mathrm{~s}$ \\
\hline
\end{tabular}

With the utilized hardware listed in Table I] the proposed channel sounder architecture enables real-time measurement without buffer overflows with the mentioned performance.

The motion of the robot arm causes a frequency response pattern with a repetition time of approximately $7.5 \mathrm{~s}$, as shown in Fig. 3. In contrast, the measurement time resolution of $10.24 \mu \mathrm{s}$ falls far below the time variance of the fast motion. Therefore, our proposed approach surpasses the fast time variance requirements for industrial applications with even higher demands.

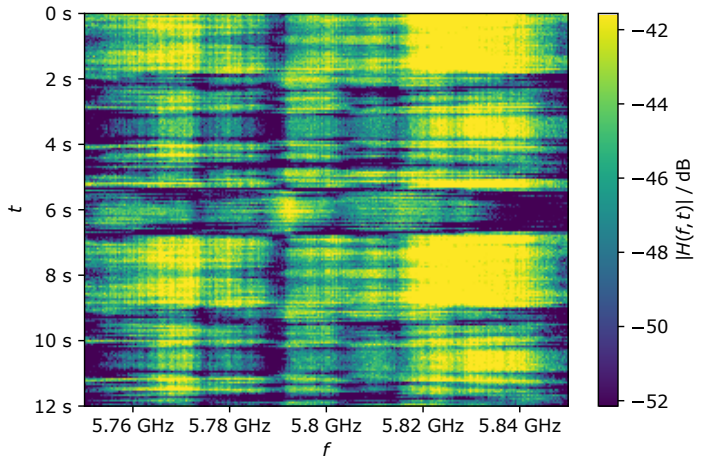

Fig. 3. Frequency response

The Doppler plot in Fig. 4 shows the impulse response and indicates the time variance of the multi-path propagation. The plot resolves distinct multi-path components in the finely granulated impulse response time resolution of 10.75 ns. Furthermore, the long-term measurement enables Doppler spread resolution of $0.083 \mathrm{~Hz}$ for time-sensitive characterization of the multi-path propagation. Additionally, the maximum resolvable Doppler spread of $48.8 \mathrm{kHz}$ corresponds to a speed of $2526 \mathrm{~m} / \mathrm{s}$.

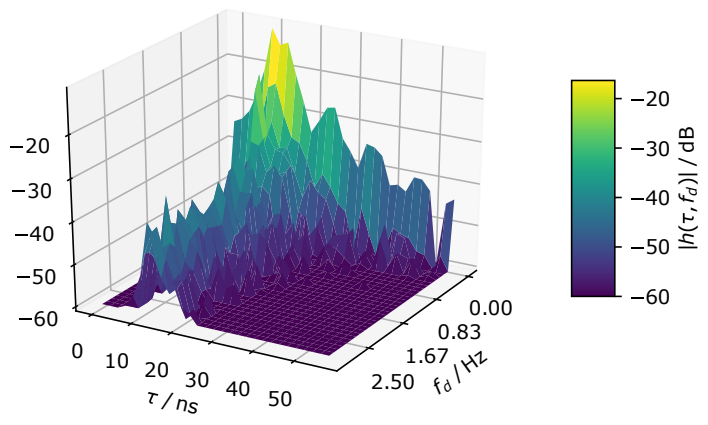

Fig. 4. Doppler plot

The application example measurement results in a dynamic range of $45.1 \mathrm{~dB}$. This dynamic range enables a measurement distance of $695.87 \mathrm{~m}$ under ideal propagation conditions.

The application example validates, that the proposed channel sounder architectures is suited for wide-band, real-time radio channel measurements with fast time variance.

\section{CONCLUSION}

Novel industrial wireless applications require wide-band, real-time channel characterization due to complex multi-path propagation. Fast time variance of the channel's reflective behavior must be captured for radio channel characterization. Additionally, inhomogeneous radio channels demand highly flexible measurement measurements. Existing approaches for radio channel measurements either lack flexibility or wideband, real-time performance with fast time variance.

The proposed approach utilizes a software-defined architecture for flexibility and scalability. Furthermore, the approach uses correlative channel sounding for real-time, wide-band 
measurements immune to active interference. Additionally, the approach enables distributed processing, and therefore it is favorable for embedded implementation.

In an application example, the distributed processing property is validated for characterization of a radio channel obstructed by robot arm motion. Hence, the proposed correlative channel sounding approach is suited for industrial wide-band, real-time channel characterization with fast time variance.

\section{ACKNOWLEDGMENT}

Parts of this work are funded by the German BMBF (project HiFlecs, 16KIS0266).

\section{REFERENCES}

[1] A. Frotzscher, U. Wetzker, M. Bauer, M. Rentschler, M. Beyer, S. Elspass, and H. Klessig, "Requirements and current solutions of wireless communication in industrial automation," in 2014 ICC - 2014 IEEE International Conference on Communication Workshop (ICC), 2014, pp. 67-72.

[2] B. Holfeld, D. Wieruch, L. Raschkowski, and T. Wirth, "Radio Channel Characterization at $5.85 \mathrm{GHz}$ for Wireless M2M Communication of Industrial Robots," in WCNC 2016 - IEEE Wireless Communications and Networking Conference, 2016.

[3] P. Stenumgaard, J. Chilo, J. Ferrer-Coll, and P. Angskog, "Challenges and conditions for wireless machine-to-machine communications in industrial environments," IEEE Communications Magazine, vol. 51, no. 6, pp. 187-192, 2013.

[4] L. Tang, K.-C. Wang, and Y. Huang, "Performance Evaluation and Reliable Implementation of Data Transmission for Wireless Sensors on Rotating Mechanical Structures," Structural Health Monitoring, vol. 8, no. 2, pp. 113-124, 2009.

[5] J. Elofsson and P. Seimar, "Software Defined Radio based MIMO channel sounding," Master's Thesis, Lund University, Lund, May 25, 2016. [Online]. Available: http://lup.lub.lu.se/luur/download?func= downloadFile \&recordOId $=8874710 \&$ fileOId $=8874711$

[6] W. Keusgen, A. Kortke, M. Peter, and R. Weiler, "A highly flexible digital radio testbed and $60 \mathrm{GHz}$ application examples," 2013.

[7] Y. Konishi, M. Kim, M. Ghoraishi, J. Takada, S. Suyama, and H. Suzuki, "Channel Sounding Technique using MIMO Software Radio Architecture," in Proceedings of the 5th European Conference on Antennas and Propagation (EUCAP). Piscataway, NJ: IEEE, 2011, pp. 2546-2550.

[8] S. Boyd, "Multitone signals with low crest factor," IEEE Transactions on Circuits and Systems, vol. 33, no. 10, pp. 1018-1022, 1986.

[9] G. Martin, "Wideband channel sounding dynamic range using a sliding correlator," in VTC 2000 - Spring. Piscataway, NJ: IEEE, 2000, pp. $2517-2521$

[10] D. Sarwate, "Bounds on crosscorrelation and autocorrelation of sequences (Corresp.)," IEEE Transactions on Information Theory, vol. 25, no. 6, pp. 720-724, 1979.

[11] H. Cao, Z. Zhao, W. Ni, M. El-Hadidy, and T. Kaiser, "Measurement and ray-tracing of wideband indoor channel in UHF TV white space," in 2011 CogART - Proceedings of the 4th International Conference on Cognitive Radio and Advanced Spectrum Management. New York, NY: ACM, 2011, pp. 1-5.

[12] J.-K. Hwang, Y.-T. Tsai, and J.-D. Li, "Multiuser channel estimation for SC-FDMA system with CAZAC sounding reference sequence and Instruments-in-MATLAB verification," in ISCE 2012 - IEEE 16th International Symposium on Consumer Electronics. [Piscataway, NJ]: [IEEE], 2012, pp. 1-5.

[13] ETSI, "LTE; Evolved Universal Terrestrial Radio Access (E-UTRA); Physical channels and modulation (3GPP TS 36.211 version 12.5.0 Release 12)," 2015.

[14] R. Svitek and S. Raman, "DC offsets in direct-conversion receivers: Characterization and implications," IEEE Microwave Magazine, vol. 6, no. 3, pp. 76-86, 2005. 